\title{
DEVELOPMENTAL CHANGES IN B-50 (GAP-43) IN PRIMARY CULTURES OF CEREBRAL CORTEX: B-50 IMMUNOLOCALIZATION, AXONAL ELONGATION RATE AND GROWTH CONE MORPHOLOGY
}

\author{
G. J. A. Ramakers,${ }^{*}$ A. B. Oestreicher,${ }^{*}$ P. S. Wolters, ${ }^{*}$ F. W. van Leeunen, ${ }^{*}$ \\ P. N. E. De Graan† and W. H. Gispen†
}

\begin{abstract}
*Netherlands Institute for Brain Research, Meibergdreef 33, 1105 AZ Amsterdam, The Netherlands and †Department of Molecular Neurobiology, Rudolf Magnus Institute, University of Utrecht, Padualaan 8, $3584 \mathrm{CH}$ Utrecht, The Netherlands
\end{abstract}

(Received 2 June 1990, in revised form 15 September 1990; accepted 1 October 1990)

\begin{abstract}
Changes in neurite outgrowth parameters and in the immunolocalization of the neuronal growth-associated protein B-50 (GAP-43) were studied in cultured neocortex as a function of development. In addition, we studied the effects of chronic blockade of bioelectric activity (BEA) with tetrodotoxin (TTX) on these parameters.

Axonal outgrowth rate in control cultures reached a maximum at 8 days in vitro (DIV) and declined to a low level at 21 DIV. B-50 staining shifted from the perikaryon to the axons and growth cones during the first 3 DIV. In axons the intensity of B-50 staining increased towards the growth cone. Within growth cones, the central/basal region and filopodia were intensely stained, whereas lamellipodia showed only marginal staining. Growth cone size gradually decreased after 3 DIV, due to the successive loss of lamellipodia and filopodia, and became club-shaped during the second week, until by 21 DIV growth cones were completely lost, and axons started retracting and degenerated. In the central area of the cultures, growth cones also decreased in size with time, but became stabilized as presynaptic elements onto other neurons.

Acute addition of TTX did not affect the outgrowth rate at 6 DIV. Chronic TTX treatment led to an earlier retraction and degeneration of axons than in control cultures and to a loss of B-50-stained cells and varicosities during the third week, but did not affect growth cone morphology or B-50 staining. The regressive phenomena are probably due to an increased neuronal cell death shown to occur after chronic TTX treatment. The developmental changes in axonal elongation rate and growth cone morphology may be related to developmental changes in the content and/or phosphorylation of B-50 (GAP-43, which are studied in the same cultures in the following paper [Ramakers et al. (1991) Int. J. Devl Neurosci. 9, 231-241].
\end{abstract}

Key words: B-50/GAP-43, cerebral cortex culture, growth cones, neurite outgrowth, bioelectric activity, tetrodotoxin.

To study neuronal development and its modulation by possible epigenetic factors we use primary cultures of dissociated fetal rat cerebral cortex..$^{35,37,52,53}$ In these cultures, the neurons rapidly form a neuronal network through neurite outgrowth and synaptogenesis ${ }^{39,52}$ and spontaneously generate action potential discharges (bioelectric activity or BEA) from the end of the first week in vitro onwards. ${ }^{17,35}$ This BEA lasts until at least 42 days in vitro (DIV), ${ }^{17,35}$ reflecting the longterm viability and functionality of the neuronal network. Recently, the cultures were adapted to be more amenable to multidisciplinary investigations, and to allow the investigation of axonal outgrowth ${ }^{36,37}$ which is an important process in neuronal network formation. Cultures of central and peripheral nervous tissue have been used extensively to investigate qualitative and quantitative aspects of neurite outgrowth (e.g. Refs $2,3,9,21,22,25$ ), but in few, if any, studies were such parameters related to biochemical processes that may underlie neurite outgrowth in the same cultures. In the present and following paper, we investigated, in the same cultures, developmental changes in various parameters of axonal outgrowth and in the localization and regulation of the B-50 protein, which has been implicated in neurite outgrowth. ${ }^{45,46} \mathrm{~B}-50$, also known as GAP-43, pp46, F1, P57 or neuromodulin, is a neuron-specific, growth-associated phosphoprotein, ${ }^{4,15,44}$ and has furthermore been shown to be involved in neuronal signal transduction, ${ }^{15.44 .48,50}$ transmitter release ${ }^{11}$ and neuronal plasticity. ${ }^{4}$ In the developing brain, B-50 is

Address correspondence to: G. J. A. Ramakers at the Department of Molecular Neurobiology, Rudolf Magnus Institute, University of Utrecht, Padualaan 8, $3584 \mathrm{CH}$ Utrecht, The Netherlands.

Abbreviations: BEA, bioelectric activity; BIR, B-50 immunoreactivity; DIV, days in vitro; TTX, tetrodotoxin. 
present in highest concentration in growth cones, ${ }^{12,47}$ where it is attached to the plasma membrane.$^{54}$ In neuronal tissue cultures, B-50 has been shown to be concentrated in growth cones, axons and the somal plasma membrane ${ }^{16,28,29,49,51}$ and to be expressed concomitant with neurite outgrowth. ${ }^{34}$

In the present study, we investigated the time course of neurite outgrowth, changes in growth cone morphology, and the localization of B-50 in cerebral cortex neurons during normal development in culture (i.e. in the presence of BEA). In the following paper ${ }^{38}$ the findings of the present study will be related to developmental changes in the regulation (content and phosphorylation) of B-50 in the same cultures. In both studies, we also investigated the effects of blocking BEA with the sodium channel blocker tetrodotoxin (TTX), which has been shown to affect neurite outgrowth $^{53}$ and synaptogenesis, ${ }^{52}$ as well as cell survival, ${ }^{35-37}$ and electrophysiological parameters $^{35}$ in cerebral cortex cultures.

\section{EXPERIMENTAL PROCEDURES}

\section{Culturing procedures and suppression of $B E A$}

Cerebral cortex from 18-19-day-old fetal rats was mechanically dissociated in chemically defined medium (CDM, see below), treated subsequently with trypsin ( $25 \mu \mathrm{g} / \mathrm{mol}$; $30 \mathrm{sec})$, Lima bean trypsin inhibitor $(50 \mu \mathrm{g} / \mathrm{mol} ; 60 \mathrm{sec})$ and DNase I ( $250 \mathrm{Kunitz}$ units $/ \mathrm{ml} ; 60 \mathrm{sec})$, after which the cells were spun down at $500 \mathrm{rpm}$ for $5 \mathrm{~min}$. After resuspension in CDM, $50 \mu \mathrm{l}$ of the cell suspension (which consisted of more than $99 \%$ living cells as assessed with trypan blue exclusion), containing 150,000 cells, was seeded onto poly-D-lysine $(10 \mu \mathrm{g} / \mathrm{ml})$ coated round coverslips (Menzelgläser, diam. $12 \mathrm{~mm}$; the whole coverslip was coated uniformly). The area that was occupied by the cells (the 'central area') was restricted to a $7 \mathrm{~mm}$ round spot using glass rings positioned in the middle of the coverslip. After $1 \mathrm{~h}$ in a $\mathrm{CO}_{2}$ incubator, two coverslips were submersed at 0 DIV into $1.5 \mathrm{ml}$ of medium in a $35 \mathrm{~mm}$ culture dish (Nunc), which contained a glial culture ('supporting glia') growing on the bottom, and the rings were removed.

Supporting glial cultures were established 12 days before by seeding 100,000 living cells from dissociated 1-2 day neonatal rat cerebral cortex in a volume of $1 \mathrm{ml}$ into the culture dishes. ${ }^{6}$ The glial cultures were grown on CDM with $10 \%$ fetal calf serum for 10 days, when medium was changed to $1.5 \mathrm{ml}$ CDM. The CDM was conditioned by the glial cells for $48 \mathrm{hr}$, whereafter two 'cerebral cortex cultures' were submersed into it. Conditioning of the medium by the supporting glial cells (mainly astrocytes) was found to be essential for long-term neuronal survival and high levels of spontaneous bioelectric activity (BEA), and yields mixed neuron-glia cultures, containing about $15-20 \%$ neurons. $^{36,37}$

Cultures were maintained at $35.5^{\circ} \mathrm{C}, 5 \% \mathrm{CO}_{2}$ and at $100 \%$ humidity, and were refreshed twice a week from 6 DIV onwards. The CDM was composed of 3 parts DMEM and 1 part Ham's F12 (without glutamate and aspartate, and containing $2 \mathrm{mM}$ calcium, $2 \mathrm{mM}$ magnesium and $3 \mathrm{mM}$ potassium; Gibco). This basal medium was supplemented with bovine serum albumin (4 g/l), insulin $(10 \mathrm{mg} / \mathrm{l})$, transferrin $(10 \mathrm{mg} / \mathrm{l})$, tocoferol $(10 \mathrm{mg} / \mathrm{l})$, retinol $(1 \mathrm{mg} / \mathrm{l})$ and ascorbic acid (10 mg/l).

Addition of TTX $(0.1 \mu \mathrm{M})$ from 1 or 6 DIV onwards was used to chronically suppress all action potential discharges. ${ }^{52}$ Parallel control cultures from the same series (coded NG93, NG95 and NG99; NG = neuron/glia) were checked for spontaneously occurring BEA during the second and third week in vitro, and all found to be active, whereas in the presence of TTX, no BEA was found. ${ }^{35}$

\section{Measurement of the width of the peripheral fiber outgrowth area}

After immunoperoxidase staining of the cultures with anti-B-50 immunoglobulins (IgGs; see below), camera lucida drawings were made of the circumference of the peripheral axonal area and the central area, making use of the higher staining intensity of the peripheral area at all ages. Since both circumferences were nicely circular and concentric in all cultures, the average width could be determined by aligning them with a set of concentric circles. 


\section{Axons elongation rate measurements}

To study axonal elongation rates, cells were grown on $0.1 \mathrm{~mm}$ thick round pieces of polypropylene foil (diam. $15 \mathrm{~mm}$ ) onto which a grid of $300 \mu \mathrm{m}$ squares was 'stamped' using a BBpress (Mecanex, Switzerland). Coating and growth conditions were similar as described above, and produced identical cultures, including cell adhesion and neurite outgrowth characteristics. Between 1 and 21 DIV, those squares in which distinct single growth cones could be clearly observed were photographed twice with an interval of 5-6 hr, using phase contrast optics. After magnification $(250 \times)$, the net increase in neurite length along the course of the axons was determined using a Calcomp 2000 digitizing tablet. The net axonal elongation rate was calculated as net increase in neurite length divided by the time interval.

\section{Immunocytochemical procedures}

Several fixatives were used to obtain optimal preservation of the fine morphology. Cultures were rinsed in phosphate-buffered saline (PBS, $10 \mathrm{mM}$ phosphate buffer, $150 \mathrm{mM}$ sodium chloride, $\mathrm{pH}$ 7.3) and then fixed in either of the following ways: (a) in acetone for $15 \mathrm{sec}$ at room temperature, (b) in $4 \%$ buffered formaldehyde for $2 \mathrm{hr}$ at $4^{\circ} \mathrm{C}$, or (c) in $5 \%$ buffered glutaraldehyde for $2 \mathrm{hr}$ at $4^{\circ} \mathrm{C}$. After fixation cultures were rinsed and stored in cacodylate buffer $(0.1 \mathrm{M}$, $\mathrm{pH} 7.3)$ at $4^{\circ} \mathrm{C}$. Acetone and formaldehyde fixed cultures were immunostained ${ }^{36}$ with rabbit-antiB-50 IgG (IgG-RH: affinity-purified from rabbit anti-B-50 serum $8613 ; 0.1 \mathrm{ng} / \mathrm{ml}^{32}$ ) as primary antibody, and affinity-purified goat anti-rabbit IgG conjugated to fluorescein isothiocyanate (Jackson Immunoresearch) as secondary antibody. Pre-immune IgG $(0.5 \mathrm{ng} / \mathrm{ml})$ was used as control. $^{32}$ Glutaraldehyde fixed cultures were immunostained for B-50, using the indirect peroxidase-anti-peroxidase (PAP) method. ${ }^{17}$

The specificity of the immunostaining procedures on the cultures has been checked according to previously established methods. ${ }^{32}$ Western blots of 21 DIV homogenates of cerebral cortex cultures, postfixed with $4 \%$ formaldehyde or $5 \%$ glutaraldehyde, and immunoincubated with anti-B-50 IgGs, revealed one staining protein band corresponding to B-50, but showed otherwise a negligible background (not shown). Immunostaining of cultures with pre-immune IgG resulted in a slight background staining of cell bodies and neurites. This background staining was mainly due to autofluorescence, since it was also observed in unstained cultures.

\section{RESULTS}

\section{Developmental changes in overall culture morphology and neurite outgrowth}

Within hours after plating, a strong outgrowth of neurites occurred, which gradually led to a dense fiber network between the cell bodies in the central area of the culture, and to a steadily increasing zone of peripheral neurites surrounding the central area (Fig. 6). In the central area of the culture, the observation of neurite outgrowth became obscured by the end of the first week, due to an increasing cell density and process outgrowth, and was only possible after PAP staining (Fig. 6). The peripheral neurites are exclusively axons, since dendrites, characterized by microtubule-associated protein 2 (MAP2) staining extend only a small distance from the cell bodies and do not reach the periphery of the culture. ${ }^{36}$ The width of this axonal zone became maximal by 14 DIV (Fig. 1a). Around 21 DIV, many peripheral axons retracted (Fig. 6f) and had completely degenerated by 35 DIV. In cultures chronically treated with TTX from 6 DIV onwards, peripheral outgrowth was similar to that seen in control cultures, but axonal retraction was accelerated by $3-4$ days. Degeneration was enhanced (Fig. 6g) and complete before 28 DIV. However, at 21 DIV, an effect of TTX was not yet apparent from the width of the axonal outgrowth area (Fig. 1a).

Quantitation of the rate of elongation of individual axons during the outgrowth period in control cultures showed a strong increase in net elongation rate between 4 and $8 \mathrm{DIV}$, followed by a strong decrease mainly during the second week (Fig. 1b). Acute addition of TTX $(0.1 \mu \mathrm{M})$ at 6 DIV did not affect the axonal elongation rate (controls: $15.1 \pm 3.0 \mu \mathrm{m} / \mathrm{hr}$; TTX-treated: $19.7 \pm 3.4 \mu \mathrm{m} / \mathrm{hr}$; both $n=21,6$ cultures; not significant at the $5 \%$ level in a two-tailed $t$ test). 


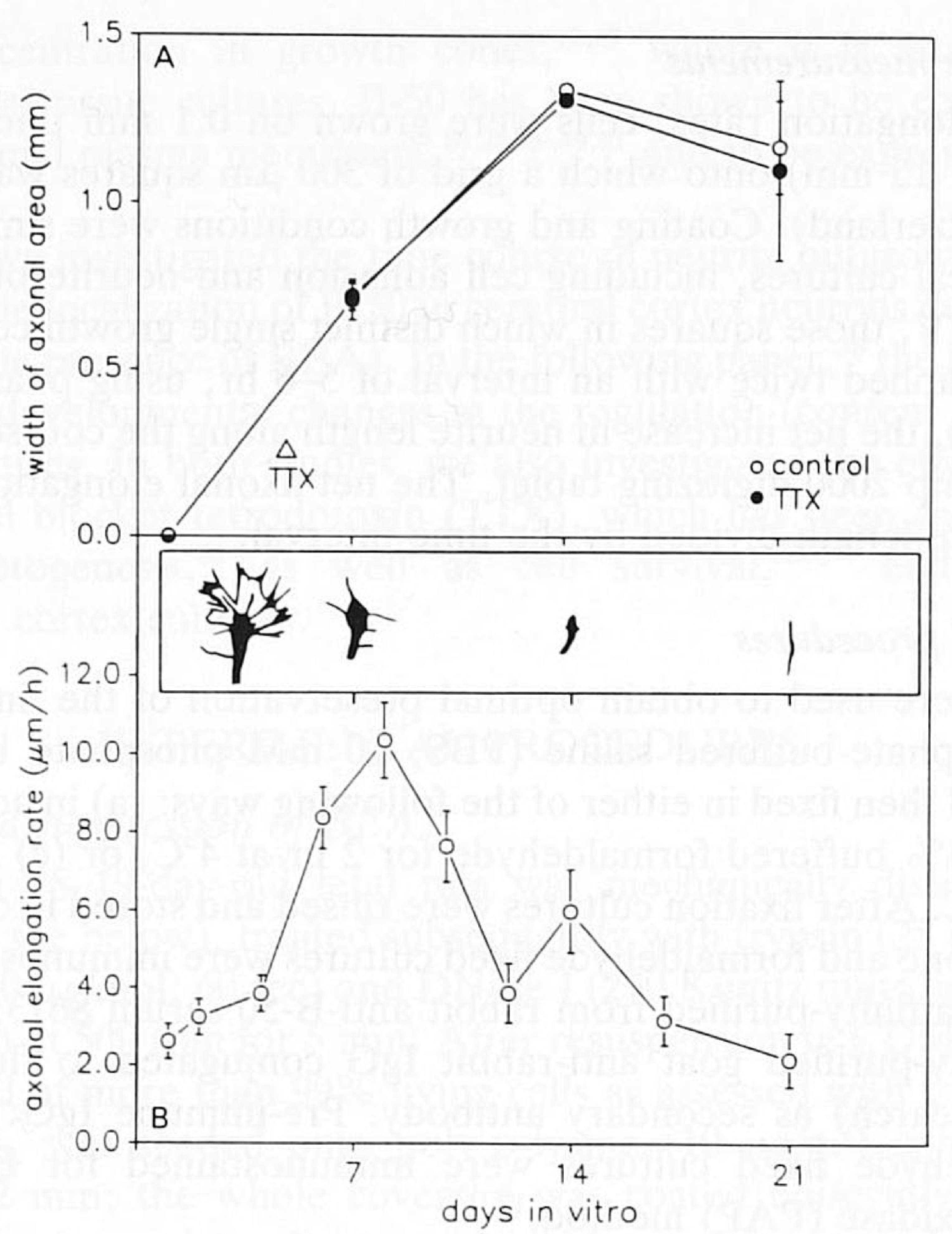

Fig. 1. Quantitation of peripheral axonal outgrowth as a function of age in vitro. A: Width of the peripheral axonal area of cultures grown in the presence or absence of TTX. Mean values \pm S.E.M.; $n=2-5$. B: Net axonal elongation rate as a function of age in vitro in control cultures. Mean values \pm S.E.M.; $n=29-67$ from 5-8 cultures between 1 and 17 DIV, and $n=9$ in 2 cultures at 21 DIV; the same cultures were followed up to 21 DIV, except for those lost due to infections. Insert: schematic summary of the developmental changes in growth cone morphology and B-50 distribution (black area) within growth cones in control cultures and cultures grown in the presence of TTX.

\section{Changes in B-50 localization and growth cone shape}

When the different fixatives were compared, formaldehyde was found to induce local swelling and shrinking artefacts, especially along axons and in growth cones. The thickness of the axons was reduced throughout, and many cytoplasmic protrusions emerged from the thinned axons and from growth cones within minutes after starting the fixation (Fig. 2). In acetone-fixed cultures, no morphological distortion was observed, whereas the intensity and distribution of B-50 immunoreactivity (BIR) were the same as in formaldehyde-fixed cultures. Glutaraldehyde fixation induced almost negligible morphological changes. Therefore, all photographs shown are taken from acetone-fixed cultures, except Fig. 6, which was derived from glutaraldehyde-fixed cultures.

Within $5 \mathrm{hr}$ after adhesion, most cells generated short neurites with growth cones, which differentiated into morphologically distinct axons and dendrites within $24 \mathrm{hr}$ (Fig. 3a, b). BIR was mainly present in the perikarya, but was also prominent in the earliest growth cones (Fig. 3a, b).

During the first 3 days, axons greatly increased in length and usually broadened towards the distal end (Figs 3c, 4a). Many growth cones elaborated extensive lamellipodia (flat, veil-like structures) and thin filopodia (microspikes), attaining maximal size at 2-3 DIV (Figs 3c-e, 4a). BIR in the perikaryal cytoplasm gradually decreased. Along axons, a gradient in BIR became established (Fig. 4a) which attained its highest levels in the basal/central area of the growth cones (Fig. 3c, d). Within growth cones, filopodia were always intensely stained (Figs 3c-e, Fig. 4), whereas lamellipodia usually were faintly stained (Fig. 3e) or only showed BIR in their marginal zone (Fig. 3c, d). Filopodia which extended laterally from many axons ('lateral filopodia'), also showed strong BIR (Fig. 3c, 4a).

Between 3 and $7 \mathrm{DIV}$, punctate staining appeared in the thin proximal parts of the axons (Fig. $4 \mathrm{~b})$. Growth cone size gradually decreased, due to the loss of all lamellipodia and part of the filopodia (Fig. 4a-d). Intense BIR now occurred throughout the mainly 'club-shaped' growth 

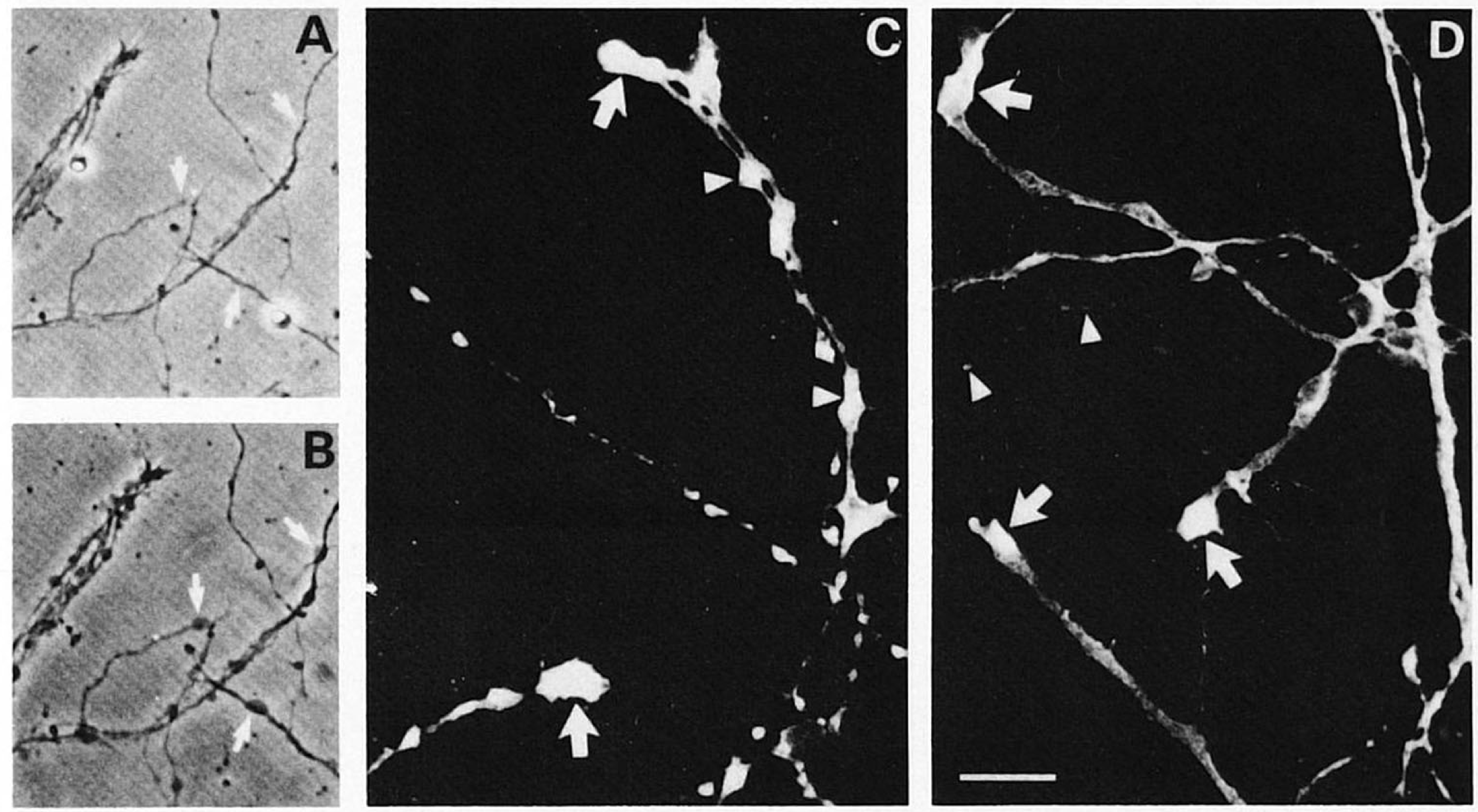

Fig. 2. Induction of morphological artefacts in axons and growth cones by formaldehyde fixation at 7 DIV. A, B: Axonal morphology seen with phase contrast optics before (A) and 5 min after (B) addition of formaldehyde fixative, showing the appearance of large varicosities (arrows). C, D: As a result, axonal B-50 staining becomes much more varicose (C) than after acetone-fixation (D) (varicosities: arrowheads; growth cones: arrows). Bar $=10 \mu \mathrm{m}$. 

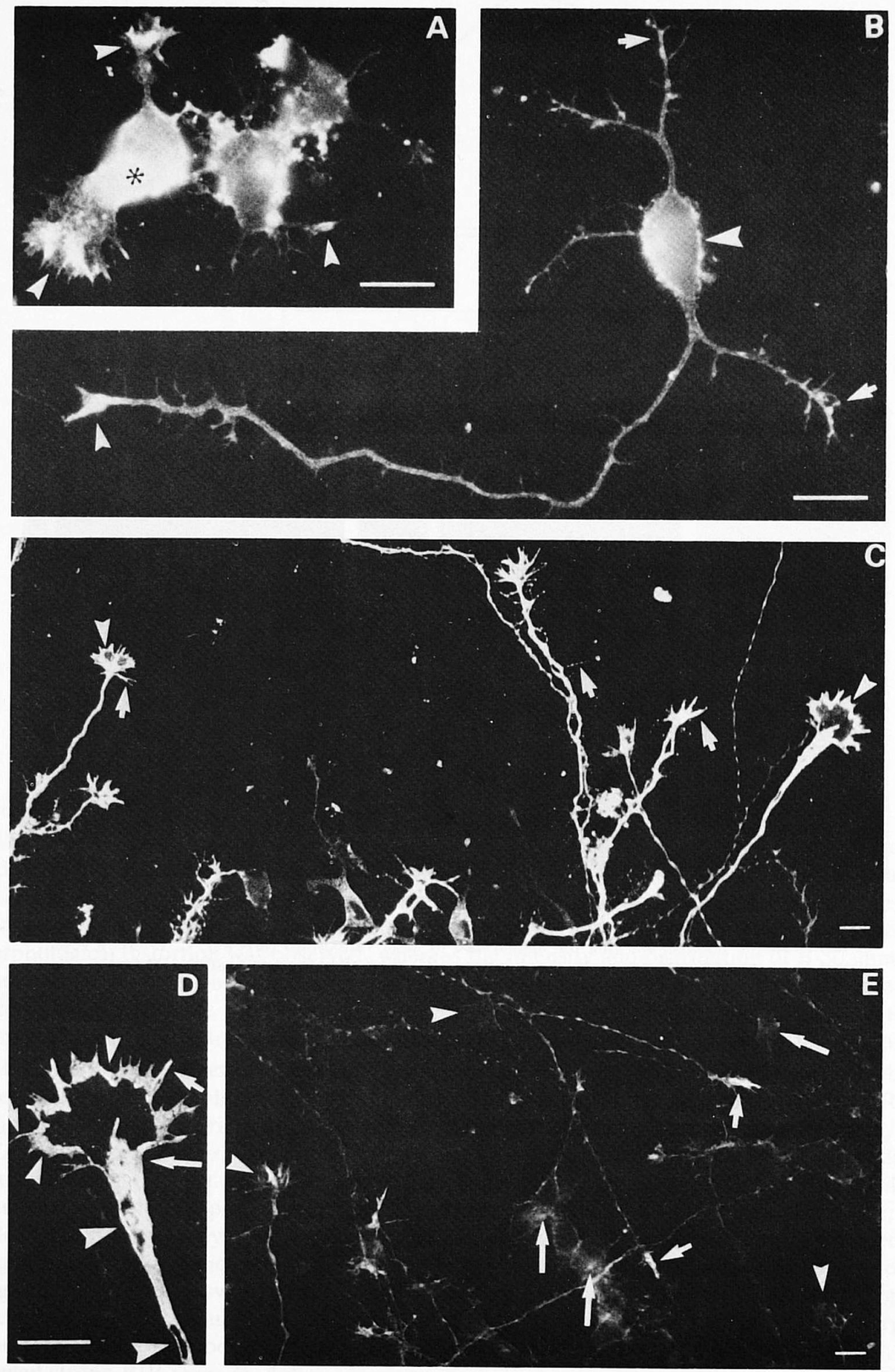

Fig. 3 (legend on page 225). 

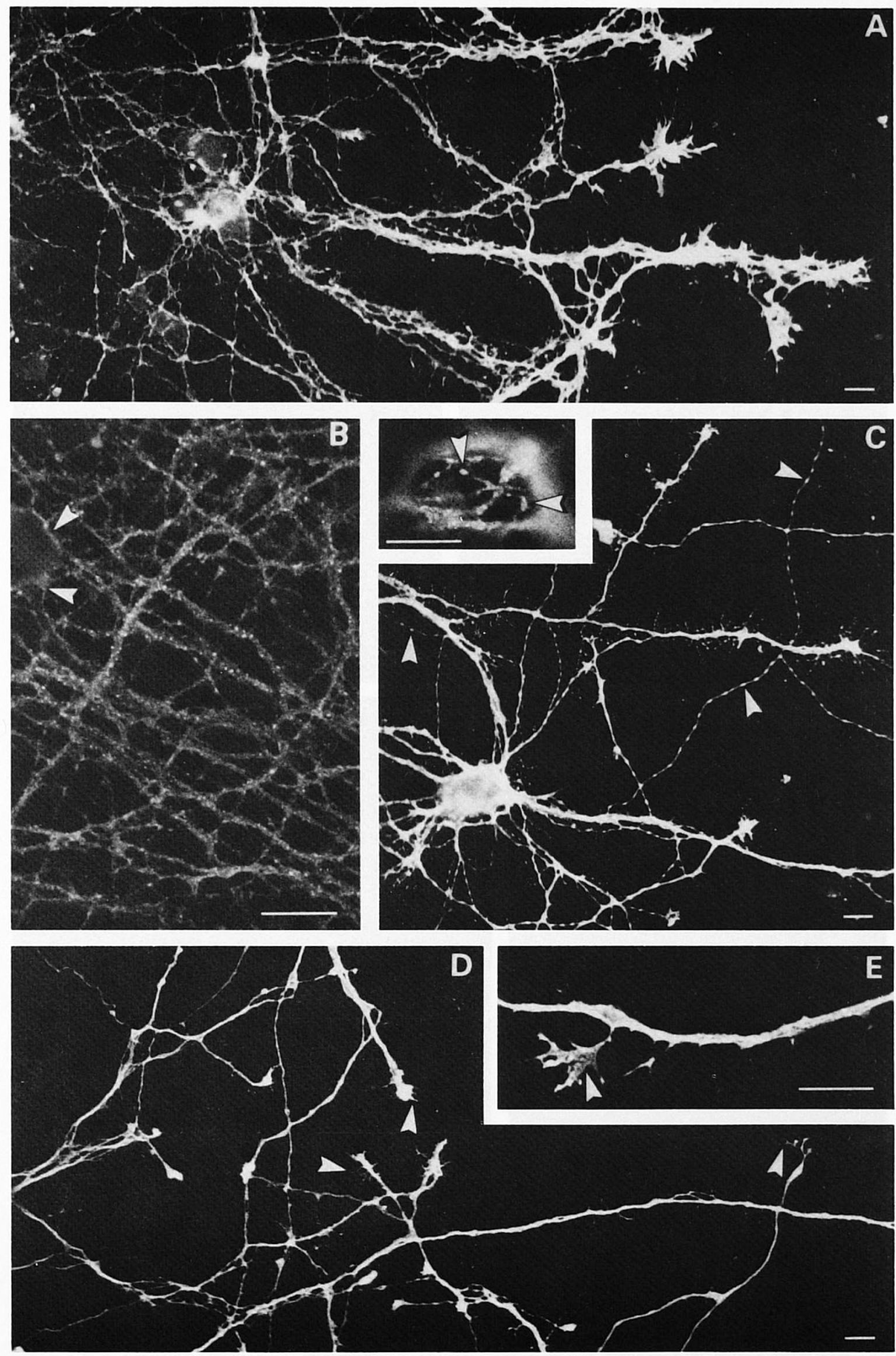

Fig. 4 (legend on page 225). 

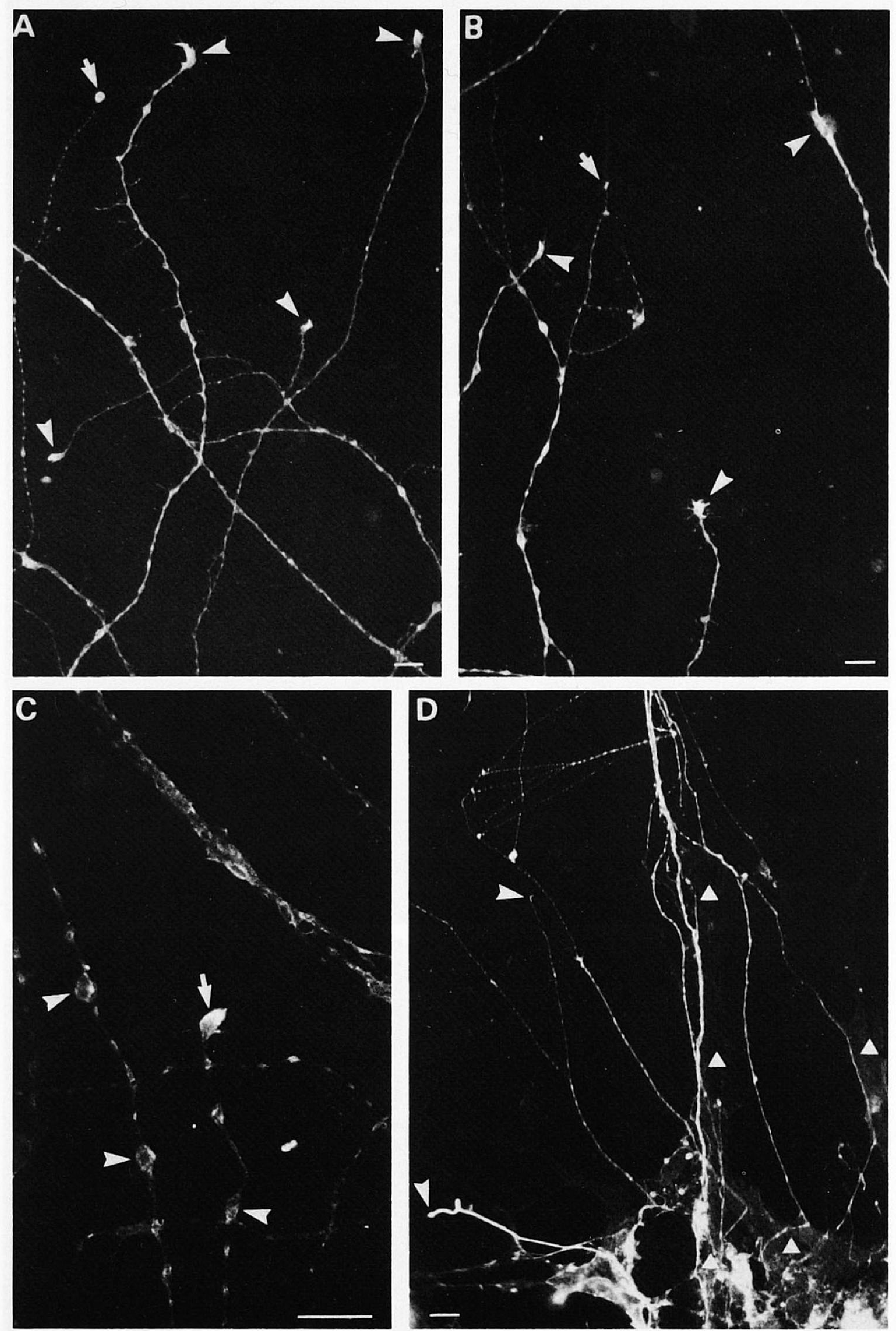

Fig. 5 (legend on page 225). 


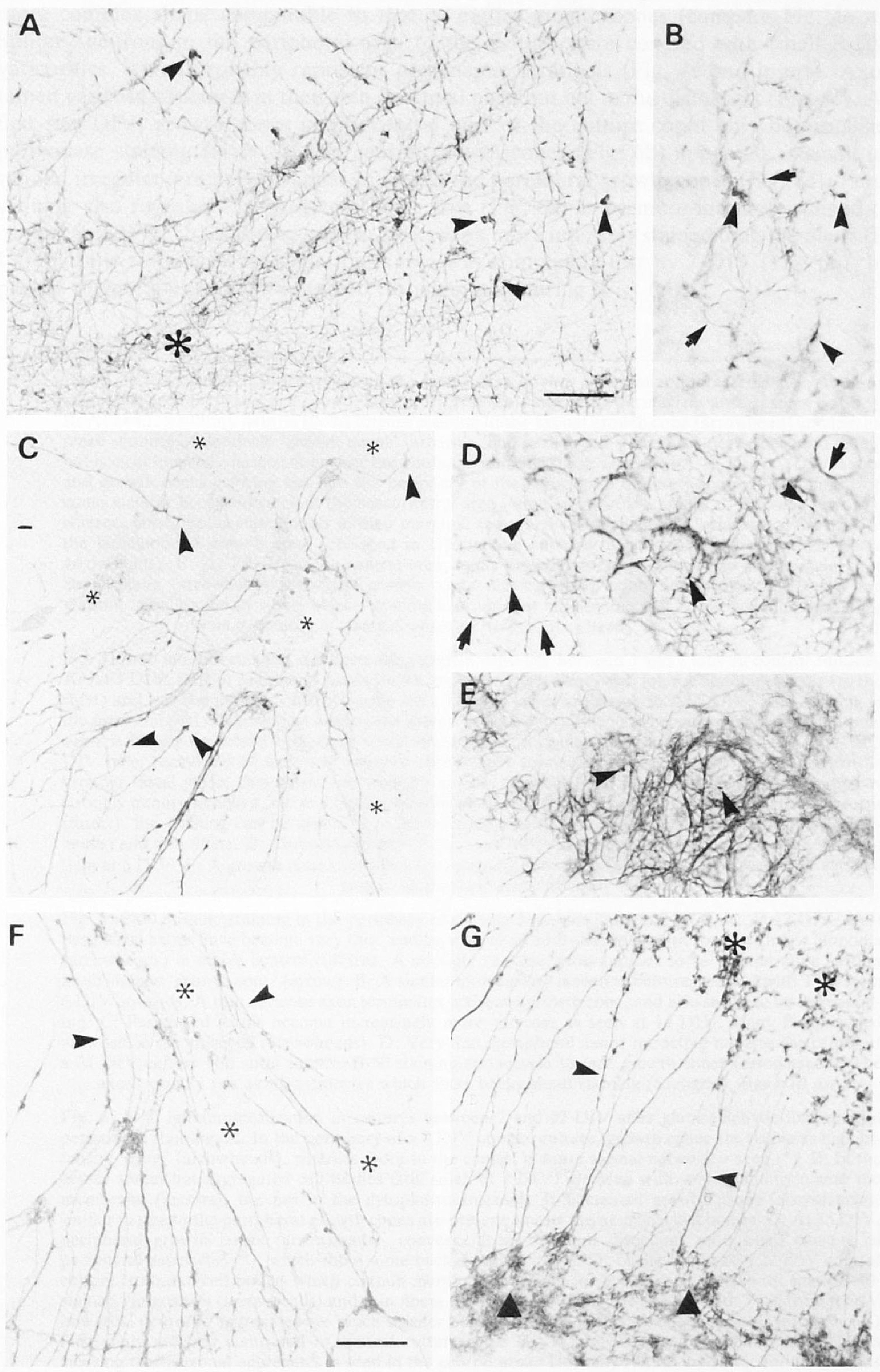

Fig. 6 (legend on page 225). 

cones (Fig. 4c, d). Occasionally, a small growth cone was elaborated along an axon, apparently from a lateral filopodium (Fig. 4e). Interestingly, such lateral growth cones usually showed a more complex shape comparable to that of earlier growth cones (compare Fig. 4e with 4a). Solitary neurons in the peripheral area of the culture were covered with small B-50-stained varicosities, which probably represent presynaptic terminals (Fig. 4c and insert). Axons contained varicose structures in their thin proximal part, but not in the distal part (Fig. 4c). After the first few DIV, growth cones in the central part of the culture could only be visualized after peroxidase staining for B-50. The central growth cones (Fig. 6b) appeared as small intensely stained irregular structures, similar in size to the peripheral growth cones (Fig. 6a). Peroxidasestaining also revealed the peripheral fiber area (Fig. 6a) to be more intensely stained than the central area (Fig. 6b), with the growth cones even more intensely stained than the fibers (Fig. 6a). Cytoplasmic staining of neuronal perikarya was completely lost by 7 DIV (Fig. 6b), but their plasma membranes remained stained throughout culturing (Fig. 6d).

Fig. 3. B-50 immunolocalization during the first 2 days in vitro by immunofluorescence. A: At $12 \mathrm{hr}$ staining is prominent in some growth cones (arrowheads), and in the perinuclear area of some cells $\left({ }^{*}\right)$. B: At $24 \mathrm{hr}$, a solitary neuron shows intense staining in the axonal growth cone (arrowhead) and less intense staining of dendritic 'growth cones' (arrows). The perikaryon shows some cytoplasmic staining, but is most intensely stained in or near the plasma membrane (large arrowhead). C, D: At 2 DIV, axons and growth cones growing out into the periphery of the culture are intensely stained. Within growth cones staining is concentrated in the basal/central area (large arrow in D), and in all filopodia (arrows), whereas lamellipodia stain mainly in their marginal zone (arrowheads). In the distal part of the axon of the lamellipodial growth cone (enlarged in D) staining appears to be displaced by organels (large arrowheads). E: At 2 DIV, in the central area, many growth cones are very broad and contain large lamellipodia (arrowheads), whereas growth cones moving along other neurites are elongated and without lamellipodia (arrows). Again staining is prominent in filopodia and low in lamellipodia. Some somal staining is also still apparent (large arrowhead). Bar $=10 \mu \mathrm{m}$.

Fig. 4. B-50 immunostaining and decreasing growth cone size between 3 and 7 DIV in control cultures. A: At 3 DIV, BIR in peripheral axons shows an intensity gradient, with intense staining distally (to the right) and low staining proximally (to the left). Growth cones are large. B: At 5 DIV, B-50 staining in the proximal part of axons (just outside the central area of the culture) has become punctate. A neuronal soma is largely unstained except for some membranous staining (arrowheads). C: Growth cones at 5 DIV have decreased in size, and become club-shaped containing filopodia only. In contrast to the broader distal parts, thin axons are strongly beaded (arrowheads). The neuronal cell body appears strongly immunoreactive, but at a higher magnification, and with the upper part of the cell body in focus (insert), the staining can be observed to derive from small B-50-immunoreactive varicosities (arrowheads) and thin fibers. D: Club-shaped growth cones at 7 DIV, containing fewer filopodia (arrowheads) than at 5 DIV. E: A growth cone (arrowhead) emanating laterally from an axon, seems to have evolved from a filopodium. Bar $=10 \mu \mathrm{m}$.

Fig. 5. B-50 immunostaining in the periphery of cultures between 12 and 21 DIV. A: At 12 DIV, most peripheral axons have become very thin, ending with small club-shaped growth cones with few filopodia (arrowheads) in active control cultures. A strongly varicose axon appears to be degenerating, with a knob-shaped 'growth cone' (arrow). B: A similar morphology is seen in cultures treated with TTX from 6 DIV onwards. A thin varicose axon terminates without a growth cone, and also seems to be degenerating. C: Peripheral axons become increasingly more varicose as seen at 14 DIV. Many B-50-stained varicosities are enlarged (arrowheads). D: Very thin, peripheral axons retracting towards the center of a 21 DIV culture still show intense B-50 staining and appear to lack growth cones (arrowheads). The axons usually run along astrocytes which show background staining (triangles). Bar $=10 \mu \mathrm{m}$.

Fig. 6. B-50 immunolocalization in cultures between 7 and 22 DIV after glutaraldehyde fixation and peroxidase staining. A: In the periphery of a 7 DIV control culture, growth cones are visible as high intensity 'spots' (arrowheads), whereas more to the center, a dense axonal network is seen $\left({ }^{*}\right)$. B: In the center somewhat aggregated cell bodies (still small at 7 DIV) are seen with some staining in/near the membrane (arrows), but not in the cytoplasm. Intensely B-50-stained growth cones (arrowheads), similar in size to the peripheral growth cones are present among the neuronal cell bodies. C: At 15 DIV, peripheral growth cones (arrowheads), converge from different directions onto small clusters of peripheral astrocytes $\left({ }^{*}\right)$, which show some background staining. D: In the center of a 22 DIV control culture, rounded cell bodies which contain membrane staining (arrows) are invested with small B-50stained varicosities (arrowheads) and thin fibers. E: In a 22 DIV culture treated with TTX from 6 DIV onwards, neuronal aggregates are much smaller. The density of B-50-stained varicosities (arrowheads) is strongly reduced compared to control cultures (see D). A high density of immunostained fibers crossing the neuronal aggregates is seen in the central area. This may reflect the retraction of neurites towards the cell bodies. F: In the periphery of a 22 DIV control culture, axons (arrows) retract along astrocytes $\left({ }^{*}\right)$. G: axonal degeneration $\left({ }^{*}\right.$ : debris) and retraction (arrowheads) in the periphery of a 22 DIV culture treated with TTX from 6 DIV onwards (compare with F). The central area also contains immunoreactive debris (triangles). The scale bar in $A$ and $G$ is $20 \mu \mathrm{m}$, and is the same for all subfigures. 
During the second week axons became thinner and progressively more beaded, with enlarged B-50-stained varicosities, and lost most of their lateral filopodia (Fig. 5a, c). Growth cones further decreased in size and lost most of their filopodia (Fig. 5a, c).

During the third week, almost all peripheral axons (showing high intensity BIR) gradually lost their growth cones (Fig. 5d, 6f). Most axons grew preferentially towards clusters of astrocytes (Fig. 6c). After contacting the astrocytes many growth cones showed little further advance, and apparently became stabilized on them (Fig. 6c). Central growth cones had also decreased in size, and seemed to have contacted neuronal somata (Fig. 6d), resembling immunostained presynaptic contacts. ${ }^{17}$ Neuronal somata showed B-50-stained plasma membranes, but no cytoplasmic staining (Fig. 6d).

After $21 \mathrm{DIV}$, daily inspection of the same areas in the peripheral area revealed that peripheral axons gradually retracted towards the central area (Figs 5d, 6f). This retraction led to increasingly larger areas along the edge of the central area without axons. Often the retracting axons appeared tightly strung. Especially in the outer peripheral area axons started to degenerate, and by 35 DIV all axons had degenerated, leaving behind B-50-positive debris where the axons once had been (as in Fig. 6g).

In the presence of TTX from 6 DIV onwards, B-50 localization and growth cone morphology showed the same development as in control cultures (compare Fig. 5a, b). However, at 21 DIV, the number of neurons (B-50-positive cells) in the central area was reduced, and TTX-treated cells contained fewer small varicosities on their perikarya (cf. Fig. 6d, e). Moreover, TTX-treated cultures showed an earlier and enhanced retraction and degeneration of the stained axons (Fig. $6 \mathrm{~g}$ ), which did not markedly affect the width of the peripheral axonal area yet at 21 DIV (Fig. 1a). TTX-treatment during the first week only led to a decreased size of neuronal (B-50-stained) cell bodies at 7 DIV (not shown).

\section{DISCUSSION}

\section{Neurite outgrowth and growth cone morphology}

Peripheral neurite outgrowth in our cultures occurred mainly during the first 2 weeks (Fig. 1), in agreement with previous observations. ${ }^{52}$ The net axonal elongation rate was low during the first four DIV, but then increased rapidly to a maximum at 8 DIV. Meanwhile, growth cones attained a maximal size around 3 DIV and then gradually became smaller again, mainly due to the loss of lamellipodia (Fig. 1). During the second week, the elongation rate decreased rapidly, accompanied by a loss of growth cone filopodia. During the third week elongation rates declined somewhat more, and growth cones were lost altogether, followed by a massive retraction and degeneration of peripheral axons. A similar developmental pattern in axonal elongation rate has been observed in other cultures. ${ }^{2,3}$ In cultured sympathetic neurons, a developmental decrease of growth cone size was found to be accompanied by a decreasing neurite elongation rate. ${ }^{3}$ We found such a parallel only after 7 DIV. However, the low net elongation rate during the first four DIV could have resulted from a high incidence of growth cone retractions in early cultures (Ref. 3, and Ramakers and Wolters, unpublished time-lapse observations). Moreover, lamellipodial growth cones have been shown to have a relatively low net elongation rate in spite of a high motility, ${ }^{27}$ possibly for the same reason.

In vivo, neocortical growth cones traveling through the corpus callosum to the contralateral neocortex, also show a strong developmental decrease in growth cone size. ${ }^{31}$ Interestingly, the size and complexity of growth cones in vivo is maximal in so-called 'decision regions' ${ }^{5,18,31}$ Thus, the complex shape of growth cones seen during the first week (i.e. filopodial or lamellipodial) as opposed to the simple (club-like) shape thereafter, could reflect a high degree of growth cone 'decision making' during the first week. Since in culture (especially in the peripheral area) most of the normal environmental cues are absent, the changes in growth cone shape, size and axonal elongation rate would seem to be largely under endogenous control. However, after the first week peripheral astrocytes could provide some environmental cues.

In the central area of the culture, growth cones were visualized by peroxidase staining for B-50. Like the peripheral growth cones, they decreased in size, but rather than being lost, central growth cones were transformed into B-50-stained presynaptic elements, ${ }^{14}$ in correspondence with 
the rise in synaptic density seen previously in these cultures. ${ }^{52}$ The persistence of the central fiber network and synapses up to 6 weeks ${ }^{36,52}$ is in strong contrast with the loss of all growth cones and axons in the peripheral area of control cultures. Most likely the loss of peripheral fibers is due to the absence of an appropriate stabilizing target ${ }^{33}$ which might become essential for the cultured neurons during the third week. Elimination of axons has also been observed with neocortical neurons in vivo. ${ }^{20,33}$ Our observations suggest that both retraction and degeneration play a role in axonal elimination in culture. The retractive forces may be quite strong as seen in time-lapse studies (Ramakers and Wolters, unpublished observations), where reaggregated clumps of neocortex neurons were found to be pulled together by tensions generated in the connecting axonal bundles. Possibly similar processes may function in axonal elimination in vivo.

The decline in axonal elongation rate and the loss of filopodia after 8 DIV coincides with increasing levels of BEA after 7 DIV in these cultures. ${ }^{17,35}$ Since electric stimulation has been shown to decrease neurite outgrowth rate,${ }^{10}$ our observations suggest that increasing levels of BEA in our cultures could be responsible for the decreasing elongation rate. However, since neither acute nor chronic TTX treatment after 6 DIV (which reliably blocks all action potential discharges ${ }^{52}$ ) markedly affected axonal outgrowth, axonal elongation rate in our cultures seems independent of BEA. Similarly, the developmental changes in growth cone morphology appear independent of BEA. Although previously TTX treatment $(1 \mu \mathrm{M})$ was found to enhance total neurite outgrowth during the first two DIV,$^{53}$ TTX treatment during the first week did not reveal such effects in the present study. This failure may be due to the use of a lower concentration of TTX $(0.1 \mu \mathrm{M})$ or different culture conditions in the present study. Our present results support the view of Schmidt and Tieman ${ }^{41}$ that chronic TTX treatment does not have a growth-promoting effect on axons in vertebrates. In addition, a recent detailed study on retinal axons regenerating in the presence or absence of TTX in vivo also revealed no differences between these two conditions. ${ }^{19}$

Morphological effect of chronic TTX treatment started at 6 DIV, were only observed after 14 DIV, and included an earlier retraction of peripheral axons and a strong reduction in the density of B-50-stained varicosities in the central area of the culture at 21 DIV. The latter observation agrees with a reduction of synaptic density as a result of chronic TTX treatment in similar cultures. ${ }^{52}$ These effects seem due to an increase in neuronal cell death, which is preceded by axonal degeneration as was previously observed in these cultures, ${ }^{36,37}$ and was now obvious from the decreased neuronal density. Similar effects of chronic TTX treatment on neuronal survival have also been found in other CNS tissue culture models. ${ }^{8,23,40}$

\section{B-50 immunolocalization}

The present finding of B-50 to be concentrated in growth cones and axons is in agreement with previous studies on neuronal cultures. ${ }^{16,28,29,42,49,51}$ However, in these studies formaldehyde was used as the main fixative, which distorts neurite and growth cone morphology, ${ }^{22}$ and results in a more varicose localization of B-50 in these structures. With acetone fixation, BIR was found to be concentrated in the basal/central area of growth cones, in all filopodia, and low in lamellipodia, except for their marginal zone. This localization resembles the submembraneous localization of actin filaments in filopodia and within the leading edge of the growth cones and in the axonal submembrane area. ${ }^{21,22,43} \mathrm{~A}$ similar sub-growth cone localization has been reported for chicken B-50, which moreover copurifies with the actin-containing neuronal submembrane cytoskeleton. ${ }^{30}$ Thus, a colocalization of $\mathrm{B}-50$ and the microfilament system is suggested. The prominent presence of B-50 in filopodia furthermore supports a role for B-50 in signal transduction, ${ }^{15,44,50}$ since filopodia are regarded to act as growth cone sensors. ${ }^{7,25,26}$

The punctate staining pattern in the proximal axon appearing at 5 DIV may point to the presence and possible transport of B-50 in membrane bound structures (e.g. clusters of vesicles, multivesicular bodies and lysosomes ${ }^{51}$ ). Likewise, the prominence of B-50 in the basal/central growth cone area corresponds to the concentration of vesicles in this area, ${ }^{9}$ which would suggest that also in growth cones B-50 could play a role in exocytosis, as found in presynaptic terminals. ${ }^{11}$ In growth cones, this exocytosis might primarily be important for adding and/or recycling plasma membrane, ${ }^{7}$ although growth cones have also been shown to release neurotransmitters. ${ }^{24}$ At present, ultrastructural investigations are being carried out to confirm the possible interactions between B-50, microfilaments and growth cone vesicles. 
During the first week, B-50 staining disappeared from the somal cytoplasm as observed in other neuronal cultures. ${ }^{28,29,49}$ However, its persistence in or near the somal plasma membrane up to at least 21 DIV seems in contrast with observations in cultured spinal neurons, which loose all somal B-50 by $3-4$ DIV. ${ }^{49}$ This difference may reflect regional differences as seen in vivo, ${ }^{13}$ or result from differences in culture conditions. The persistence of B-50 in retracting and even regenerating axons suggests that the mere presence of B-50 is not important for stabilizing the axons. Moreover, the accelerated retraction and degeneration of peripheral axons upon chronic TTX treatment was not accompanied by an altered distribution of B-50 in these axons. However, these regressive phenomena may have resulted from changes in the regulation of B-50, which will be addressed in the following paper. ${ }^{38}$

\section{CONCLUSIONS}

Developmental changes in axonal elongation rate were accompanied by changes in growth cone morphology and size, but not by changes in B-50 distribution in cultured neocortex. Moreover, all these developmental changes were independent of BEA, which did however, affect axonal retraction and neuronal survival. The sub-localization of B-50 within growth cones could support a role for B-50 in exocytosis and/or membrane plasticity as well as in signal transduction. In the following paper ${ }^{38}$ we will investigate whether the developmental changes in neurite outgrowth and morphology in these cultures are reflected in biochemical changes of B-50.

Acknowledgements-The authors wish to thank R. J. Bloemen for the purification of the B-50 antibodies, E. D. Kluis and G. van der Meulen for art work and photography, and Dr G. J. Boer for his valuable criticisms.

\section{REFERENCES}

1. Allsop T. E. and Moss D. J. (1989) A developmentally regulated chicken neuronal protein associated with the cortical cytoskeleton. J. Neurosci. 9, 13-24.

2. Argiro V. and Johnson M. I. (1982) Patterns and kinetics of neurite extension from sympathetic neurons in culture are age dependent. J. Neurosci. 4, 503-512.

3. Argiro V., Bunge M. B. and Johnson M. I. (1984) Correlation between growth cone form and movement and their dependence on neuronal age. J. Neurosci. 12, 3051-3062.

4. Benowitz L. I. and Routtenberg A. (1987) A membrane phosphoprotein associated with neural development, axonal regeneration, phospholipid metabolism and synaptic plasticity. Trends Neurosci. 12, 527-532.

5. Bovolenta P. and Mason C. A. (1987) Growth cone morphology varies with position in the developing mouse visual pathway from retina to first targets. J. Neurosci. 7, 1447-1460.

6. Booher J. and Sensenbrenner M. (1972) Growth and cultivation of dissociated neurons and glial cells from embryonic chick, rat and human brain in flask cultures. Neurobiology 2, 97-105.

7. Bray D. and Hollenbeck P. J. (1988) Growth cone motility and guidance. Ann. Rev. Cell Biol. 4, 43-61.

8. Brenneman D. E., Neale E. A., Habig W. H., Bowers L. M. and Nelson P. G. (1983) Developmental and neurochemical specificity of neuronal deficits produced by electrical impulse blockade in dissociated spinal cord cultures. Devl Brain Res. 9, 13-27.

9. Burmeister D. W., Chen M., Bailey C. H. and Goldberg D. J. (1988) The distribution and movement of organelles in maturing growth cones: correlated video-enhanced and electron microscopic studies. J. Neurocytol. 17, $783-795$.

10. Cohan C. S. and Kater S. B. (1986) Suppression of neurite elongation and growth cone dynamics by electrical activity. Science 232, 1638-1640.

11. Dekker L. V., De Graan P. N. E., Oestreicher A. B., Versteeg D. H. G. and Gispen W. H. (1990) Inhibition of noradrenaline release by antibodies to B-50 (GAP-43). Nature 342, 74-76.

12. De Graan P. N. E., Van Hooff C. O. M., Tilly B. C., Oestreicher A. B., Schotman P. and Gispen W. H. (1985) Phosphoprotein B-50 in nerve growth cones from fetal rat brain. Neurosci. Lett. 61, 235-241.

13. De la Monte S. M., Federoff H. J., Ng S.-C., Grabcyk E. and Fishman M. C. (1989) GAP-43 gene-expression during development: persistence in a distinctive set of neurons in the mature central nervous system. Devl Brain Res. 46, 161-168.

14. Gispen W. H., Leunissen J. L. M., Oestreicher A. B., Verkleij A. J., Zwiers H. (1985) Presynaptic localization of B-50 phosphoprotein: the ACTH sensitive protein kinase substrate involved in rat brain polyphosphoinositide metabolism. Brain Res. 328, 381-385.

15. Gispen W. H., Van Dongen C. J., De Graan P. N. E., Oestreicher A. B. and Zwiers H. (1985) The role of phosphoprotein B-50 in phosphoinositide metabolism in brain synaptic plasma membranes. In Inositol and Phosphoinositides (eds. Bleasdale J. E., Hauser G. and Eichberg J.), pp. 399-413. Humana Press, New York.

16. Goslin K., Schreyer D. J., Skene J. H. P. and Banker G. (1988) Development of neuronal polarity: GAP-43 distinguishes axonal from dendritic growth cones. Nature 336, 672-674.

17. Habets A. M. M. C., Van Dongen A. M. J., Van Huizen F. and Corner M. A. (1987) Spontaneous neuronal firing patterns in fetal rat cortical networks during development in vitro: a quantitative analysis. Exp. Brain Res. 69, 43-52. 
18. Harris W. A., Holt C. E. and Bonhoeffer F. (1987) Retinal axons with and without their somata growing to and arborizing in the tectum of Xenopus embryos: a time-lapse video study of single fibres in vivo. Development 101, 123133.

19. Hartlieb E. and Stuermer C. A. O. (1989) Pathfinding and target selection of goldfish retinal axons regenerating under TTX-induced impulse blockade. J. Comp. Neurol. 284, 148-168.

20. Innocenti G. M. (1981) Growth and reshaping of axons in the establishment of visual callosal connections. Science 212, 824-827.

21. Isenberg G. and Small I. V. (1978) Filamentous actin, 100 A filaments and microtubules in neuroblastoma cells. Their distribution in relation to sites of movement and neuronal transport. Eur J. Cell Biol. 16, 326-344.

22. Letourneau P. C. (1981) Immunocytochemical evidence for colocalization in neurite growth cones of actin and myosin and their relationship to cell-substratum adhesions. Devl Biol. 85, 113-122.

23. Lipton S. A. (1986) Blockade of electrical activity promotes the death of mammalian retinal ganglion cells in culture. Proc. Natl. Acad. Sci. U.S.A. 83, 9774-9778.

24. Lockerbie R. O. and Gordon-Weeks P. R. (1986) Further characterization of $\left[{ }^{3} \mathrm{H}\right]-\mathrm{GABA}$ release from isolated neuronal growth cones: role of intracellular calcium stores. Neuroscience 17, 1257-1266.

25. Marsh L. and Letourneau P. C. (1984) Growth of neurites without filopodial or lamellipodial activity in the presence of cytochalasin B. J. Cell Biol. 99, 2041-2047.

26. Mattson M. P. (1988) Neurotransmitters in the regulation of neuronal cytoarchitecture. Brain Res. Rev. 13, $179-212$.

27. Mattson M. P. and Kater S. B. (1987) Calcium regulation of neurite elongation and growth cone motility. $J$. Neurosci. 7, 4034-4043.

28. Meiri K. F., Pfenninger K. H. and Willard M. B. (1986) Growth-associated protein, GAP-43, a polypeptide that is induced when neurons extend axons, is a component of growth cones and corresponds to pp46, a major polypeptide of a subcellular fraction enriched in growth cones. Proc. Natl. Acad. Sci. U.S.A. 83, 3537-3541.

29. Meiri K. F., Willard M. and Johnson M. I. (1988) Distribution and phosphorylation of growth-associated protein GAP-43 in regenerating sympathetic neurons in culture. J. Neurosci. 8, 2571-2581.

30. Moss D. J., Fernyhough P., Hapman K., Baizer L., Bray D. and Allsopp T. (1990) Chicken growth-associated protein GAP-43 is tightly bound to the actin-rich neuronal membrane skeleton. J. Neurochem. 54, 729-736.

31. Norris C. R. and Kalil K. (1990) Morphology and cellular interactions of growth cones in the developing corpus callosum. J. Comp. Neurol. 293, 268-281.

32. Oestreicher A. B., Van Dongen C. J., Zwiers H. and Gispen W. H. (1983) Affinity-purified anti-B-50 protein antibody: interference with the function of the phosphoprotein B-50 in synaptic plasma membranes. J. Neurochem. 41, 331-340.

33. O'Leary D. D. M. (1987) Remodeling of early axonal projections through the selective elimination of neurons and long axon collaterals. In Selective Neuronal Death (eds. Bock G. and O'Connor M.), pp. 113-142. Wiley, Chichester.

34. Perrone-Bizzozero N. I., Finklestein S. P. and Benowitz L. I. (1986) Synthesis of a growth-associated protein by embryonic rat cerebrocortical neurons in vitro. J. Neurosci. 6, 3721-3730.

35. Ramakers G. J. A., Corner M. A. and Habets A. M. M. C. (1990) Development in the absence of spontaneous bioelectric activity results in increased stereotyped burst firing in cultures of dissociated cerebral cortex. Exp. Brain Res. 79, 157-166.

36. Ramakers G. J. A., Raadsheer F. C., Corner M. A., Ramaekers F. C. S. and Van Leeuwen F. W. Development of neurons and glial cells in cerebral cortex, cultured in the presence or absence of bioelectric activity. I. Morphological observations. Eur J. Neurosci. (in press).

37. Ramakers G. J. A. and Boer G. J. Development of neurons and astrocytes in cerebral cortex, cultured in the presence or absence of bioelectric activity. II. Biochemical observations. Eur. J. Neurosci. (in press).

38. Ramakers G. J. A., De Graan P. N. E., Oestreicher A. B., Boer G. J., Corner M. A. and Gispen W. H. (1991) Developmental changes in B-50/GAP-43 in primary cultures of cerebral cortex: content and phosphorylation of B-50. Int. J. Devl Neurosci. 9, 231-241.

39. Romijn H. J., Van Huizen F. and Wolters P. S. (1984) Towards an improved serum-free, chemically defined medium for long-term culturing of cerebral cortex tissue. Neurosci. Biobehav. Rev. 8, 301-334.

40. Ruijter J. M., Baker R. E., De Jong B. M. and Romijn H. J. (1991) Chronic blockade of bioelectric activity in neonatal rat cortex grown in vitro: morphological effects. Int. J. Devl. Neurosci. (in press).

41. Schmidt J. and Tieman S. B. (1989) Activity, growth cones and the selectivity of visual connections. Comments Devl Neurobiol. 1, 11-28.

42. Schmidt-Michels M., Edwards P. M., Oestreicher A. B. and Gispen W. H. (1989) Colchicine effect on B-50/GAP43 phosphoprotein localization in rat dorsal root ganglion explants. Neurosci. Lett. 97, 285-290.

43. Shaw G., Osborn M. and Weber K. (1981) Arrangement of neurofilaments, microtubules and microfilamentassociated proteins in cultured dorsal root ganglia cells. Eur J. Cell Biol. 24, 20-27.

44. Skene J. H. P. (1989) Axonal growth-associated proteins. Ann. Rev. Neurosci. 12, 127-156.

45. Skene J. H. P. and Willard M. (1981) Changes in axonally transported proteins during axon regeneration in toad retinal ganglion cells. J. Cell Biol. 89, 86-95.

46. Skene J. H. P. and Willard M. (1981) Axonally transported proteins associated with axon growth in rabbit central and peripheral nervous system. J. Cell Biol. 89, 96-103.

47. Skene J. H. P., Jacobson R. D., Snipes G. J., McGuire C. B., Norden J. J. and Freeman J. A. (1986) A protein induced during nerve growth (GAP-43) is a major component of growth-cone membranes. Science 233, 783-786.

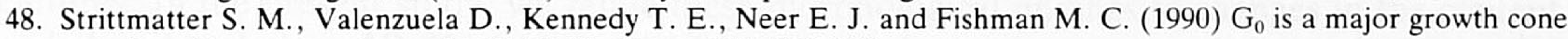
protein subject to regulation by GAP-43. Nature 344, 836-841.

49. Van der Neut R., Oestreicher A. B., Gispen W. H. and Bär P. R. (1989) The expression of B-50/GAP-43 in developing spinal neurons in cultures is regulated by interneuronal contact. Neurosci. Lett. 109, 36-41.

50. Van Hooff C. O. M., De Graan P. N. E., Oestreicher A. B. and Gispen W. H. (1988) B-50 phosphorylation and polyphosphoinositide metabolism in nerve growth cone membranes. J. Neurosci. 8, 1789-1795.

51. Van Hooff C. O. M., Boonstra J., Oestreicher A. B., De Graan P. N. E., Holthuis J. C. M. and Gispen W. H. (1989) Nerve growth factor-induced changes in the intracellular localization of the protein kinase $\mathrm{C}$ substrate B-50 in pheochromacytoma PC12 cells. J. Cell Biol. 108, 1115-1125. 
52. Van Huizen F., Romijn H. J. and Habets A. M. M. C. (1985) Synaptogenesis in rat cerebral cortex cultures is affected during chronic blockade of spontaneous bioelectric activity by tetrodotoxin. Devl Brain Res. 19, 67-80.

53. Van Huizen F. and Romijn H. J. (1987) Tetrodotoxin enhances initial neurite outgrowth from fetal rat cerebral cortex cells in vitro. Brain Res. 408, 271-274.

54. Van Lookeren Campagne M., Oestreicher A. B., Van Bergen en Henegouwen P. M. P. and Gispen W. H. (1989) Ultrastructural immunocytochemical localization of B-50/GAP-43, a protein kinase C substrate, in isolated presynaptic nerve terminals and nerve growth cones. J. Neurocytol. 18, 479-489. 